\title{
Efeitos do tipo de incubação e da forma física da ração pré-inicial sobre o desenvolvimento intestinal de pintos de corte
}

\author{
[Effects of the incubation type and the physical form of the pre-starter feed on the intestinal \\ development of broilers]
}

\section{"Revisão/Review"}

\section{José Neto Cassiano de Camargo $^{1 *}$, Eliete Souza Santana ${ }^{1}$, Denise da Costa Barboza do Carmo ${ }^{1}$, Edgar Estevam de França $^{2}$, Michele Laboissière ${ }^{3}$}

\author{
${ }^{1}$ Programa de Pós-Graduação Stricto-Sensu em Desenvolvimento Rural Sustentável, Universidade Estadual de Goiás, \\ São Luís de Montes Belos-GO, Brasil. \\ ${ }^{2}$ Programa de Pós-Graduação Stricto-Sensu em Engenharia Agrícola, Universidade Estadual de Goiás, Anápolis-GO, \\ Brasil. \\ ${ }^{3}$ Departamento de Zootecnia, Universidade Estadual de Goiás, São Luís de Montes Belos-GO Brasil. \\ *Autor para correspondência/Corresponding author: E-mail: neto_plsc@ hotmail.com
}

\begin{abstract}
Resumo
A cadeia produtiva agroindustrial brasileira é respeitada no mundo todo, pois é altamente competitiva, eficiente e possui altos índices de produtividade, havendo necessidade de aprimoramento pelo setor, o qual é possível por meio da relação entre pesquisadores e indústrias. $\mathrm{O}$ aumento dessa produção está relacionado diretamente aos aspectos nutricionais. O correto balanceamento alimentar para frangos de corte, com quantidade equilibrada de energia e aminoácidos é de fundamental importância. O manejo nutricional pode ser um fator limitante de produção, correspondendo por cerca de $70 \%$ de todos os gastos da cadeia produtiva, logo tem despertado interesse de empresas e pesquisadores. A fase de incubação dos ovos tem como objetivo, manter a produção em quantidade e qualidade satisfatórias, produzindo aves livres de micro-organismos patogênicos, com preço reduzido e com o intuito principal de aumentar e melhorar os resultados finais da produção industrial. O objetivo desta revisão é o de buscar e copilar trabalhos voltados para produção avícola, em especial, no que tange a nutrição e incubação de frangos de corte.
\end{abstract}

Palavras-chave: frangos; incubadoras; nutrição.

\begin{abstract}
The Brazilian agroindustrial production chain is respected throughout the world, as it is highly competitive, efficient and has high productivity rates, and there is a need for improvement in the sector, which is possible through the relationship between researchers and industries. The increase of this production is directly related to the nutritional aspects. Correct feed balancing for broilers with balanced amounts of energy and amino acids is of fundamental importance. Nutritional management can be a limiting factor of production, accounting for about $70 \%$ of all production chain expenditures, which has thus attracted interest from companies and researchers. The incubation phase of the eggs aims to maintain production in satisfactory quantity and quality, producing birds free of pathogenic microorganisms, with reduced price and with the main intention of increasing and improving the final results of industrial production. The objective of this review is to search for and to compile work focused on poultry production, especially in relation to nutrition and incubation of broilers.
\end{abstract}

Keywords: chickens; incubators; nutrition.

\section{Introdução}

A agropecuária brasileira é respeitada no mundo todo, pois é altamente competitiva, eficiente e possui altos índices de produtividade. $\mathrm{E}$

por essas e outras características, a mesma deve adotar várias medidas para que seu desenvolvimento ocorra de forma satisfatória, 
gerando renda, empregos e desempenhe em sua plenitude seu papel social (Perobelli et al., 2017).

Em 2017, segundo dados da Associação Brasileira de Proteína Animal - ABPA (ABPA, 2018), o Brasil produziu aproximadamente 13,05 milhões de toneladas de carne de frango, sendo destinadas ao consumidor interno cerca de $66,9 \%$ desta produção. Logo, a parte remanescente foi comercializada com países do Oriente Médio, Ásia e Europa. Para atender toda esta demanda por alimentos, os produtores devem sempre procurar otimizar suas técnicas, diminuir custos e buscar novas tecnologias (Reis et al., 2015).

O aumento dessa produção está relacionado diretamente aos aspectos nutricionais. Santos et al. (2014) e Andrade et al. (2016) analisaram que ao realizarem o balanceamento alimentar para frangos de corte, a quantidade equilibrada de energia e aminoácidos oferecidos aos animais é de fundamental importância. Já que o manejo nutricional pode ser um fator limitante de produção, pois representa alto custo, correspondendo cerca de $70 \%$ de todos os gastos da cadeia produtiva, o que tem despertado interesse de empresas e pesquisadores.

Além da qualidade nutricional das matériasprimas utilizadas na produção de ração, outro fator muito relevante é a forma física desta mistura. As aves costumam optar por um alimento com granulometria de maior tamanho, rejeitando as com espessura mais fina. Essas características são capazes de influenciar o $\mathrm{pH}$ do trato gastrointestinal, alterar a ingestão de alimentos e causar alterações metabólicas (Surek et al., 2008).

Porém, estudos relatam que a rações peletizadas com micro granulometria são aquelas que apresentam os melhores resultados produtivos ao longo de todo ciclo de vida das aves. Contudo, as mesmas são as mais caras por causa da realização da moagem mais complexa e demorada. Essas informações mostram que o tamanho da partícula de ração deve ser ideal para o animal, entretanto, deve-se levar em consideração os custos de produção (Andrade et al., 2016b).

Segundo Baracho et al. (2010), a fase de incubação dos ovos tem como objetivo, manter a produção em quantidades e qualidades satisfatórias, produzindo aves livres de microorganismos patogênicos, com preço reduzido e com o intuito principal de aumentar e melhorar os resultados finais da produção industrial. As máquinas de incubação dentro deste contexto desempenham um importante papel, pois influenciam diretamente na qualidade das aves, e atualmente, as empresas possuem opção de escolha em relação a esse processo, podendo optar por estágio múltiplo ou único, sendo que as mesmas devem levar em consideração suas excepcionalidades e capacidade financeira.

Este trabalho teve por objetivo utilizar de literaturas especializadas no ramo avícola, com ênfase para nutrição pré-inicial e incubação dos frangos de corte. Para conhecer e mostrar a importância de realizar um manejo de produção adequado, e assim, obter a máxima produtividade do animal.

\section{Rações micropeletizadas para aves}

Para fabricação de uma ração peletizada, utiliza-se primeiramente de uma ração farelada. A mesma passa por um processo mecânico e térmico, que tem como objetivo agregar as partículas dos alimentos por meio de compressão sob altas temperaturas $\left(80\right.$ a $\left.90^{\circ} \mathrm{C}\right)$ e vapor, a fim de obter péletes com grau de durabilidade suficiente para suportar as condições de transporte e manuseio. Para uma granulometria ideal, deve-se preconizar produzir grânulos com dimensões prédeterminadas que devam estar com diâmetro de $1,18 \mathrm{~mm}$ para os pintos e de $2,36 \mathrm{~mm}$ para os animais mais velhos (López e Baião, 2004; Cardeal et al., 2014).

O método de peletização já é bastante difundido nas agroindústrias e granjas brasileiras. Pelo fato de apresentar determinadas vantagens para a nutrição animal, dentre estas cabe citar a diminuição do desperdício de ração, redução da segregação de ingredientes, diminuição de microrganismos patogênicos, melhora na preferência alimentar, redução da seletividade, facilidade de apreensão da dieta, aumento da energia produtiva em função do menor tempo gasto para consumo e aumento da digestibilidade de diferentes frações da dieta (Lorençom et al., 2007).

Entre os benefícios da digestão, Lara et al. (2013) citaram que ocorre uma melhora da digestão de extrato etéreo pelas aves, em comparação com aqueles animais nutridos somente com ração farelada. Além de, possivelmente melhorar o aproveitamento dos carboidratos, isso provavelmente por causa das características de produção dos péletes. Portanto, esses fatores nutricionais comprovam que mesmo sendo mais cara que a farelada, sua utilização compensa, pois, na primeira semana, o consumo é baixo e não onera 
o custo de produção. Além de promover benefícios no desempenho até a idade de abate.

De acordo com outros autores, alguns cuidados são necessários ao utilizar rações peletizadas, pois aves com este tipo de dieta, são mais propensas a apresentarem doenças metabólicas como síndrome de morte súbita e ascite como consequências de seu alto desempenho zootécnico (López et al., 2007). O que seria justificado pelo tempo de consumo reduzido, associado a maior quantidade da ração ingerida e, consequentemente, maior ganho de peso, aumentando a demanda de oxigênio e deixando os frangos mais susceptíveis a estes tipos de doenças. Os frangos são animais muito sensíveis, sofrem com qualquer tipo de mudanças, e a escolha da configuração física do alimento deverá levar em consideração a idade, fisiologia e finalidade produtiva do animal (Melo et al., 2016).

A fase pré-inicial, na qual a ração peletizada é a mais utilizada, representa cerca de $17 \%$ de toda vida das aves, por isso, a importância de se oferecer um alimento alta qualidade se impõe, já que qualquer erro durante esta fase pode comprometer todo ciclo de produção. $\mathrm{O}$ momento de se iniciar o fornecimento de ração deve ser o mais rápido possível após a eclosão, pois é gasto um tempo considerável durante os processos de sexagem, vacinação, transporte e acomodação com os pintos ainda em jejum. Como os nutrientes do resíduo do saco da gema não são capazes de manter o desenvolvimento dos pintos nos primeiros dias de vida, podendo trazer consequências até sobre o ganho de pesos na idade de abate (Teixeira et al., 2009).

Quando se trata de rações peletizadas, alguns cuidados são necessários, pois até mesmo o transporte pode ocasionar alguns prejuízos, como diminuir quantidade de péletes íntegros em até $6 \%$. Cardeal et al. (2014) se comprometeram em avaliar as consequências da distribuição na qualidade dos péletes, e chegaram a conclusão que no momento de descarregar a ração nos silos da propriedade, deve-se manter a caixa do caminhão com a maior abertura, para não ocorrer queda na qualidade do alimento.

\section{Dietas pré-iniciais}

Os primeiros dias pós-eclosão dos pintos de corte podem ser considerados como a transição da vida embrionária e a vida independente do animal, e são de fundamental importância para todo o ciclo produtivo avícola. Preconiza-se uma dieta diferenciada nos primeiros dias de vida das aves, porque neste período ocorre uma fisiologia digestiva peculiar, principalmente na primeira semana, pois as aves não estão plenamente adaptadas à digestão de carboidratos e lipídios, sendo estes os principais fatores que revelam a necessidade de uma dieta específica e com boa qualidade e disponibilidade de nutrientes (Stringhini et al., 2003; Boemo et al., 2016). Ressalte-se que esses conhecimentos propiciaram o desenvolvimento de rações pré-iniciais.

A utilização de matérias-primas de alta qualidade e com origem certificada, se mostra como fator inafastável para produção de uma ração com quantidades apropriadas de aminoácidos e energia para as aves. Essas preocupações se mostram relevantes para sempre buscar uma melhor uniformidade dos lotes e reduzir o máximo possível a taxa de mortalidade inicial dos animais, procurando sempre otimizar o manejo alimentar, reduzindo custos de produção e permitindo que a ave expresse toda sua capacidade genética (Gomes et al., 2008).

Cada linhagem de aves possui suas características, e por implicação, suas exigências também são diferentes. O sexo, umidade do ar, estado sanitário, nível energético da ração, disponibilidade de nutrientes, consumo de ração, além de outras particularidades, são alguns dos fatores que podem influenciar as exigências nutricionais dos animais (Rostagno et al., 2011; Tavernari et al., 2014).

A alimentação animal é complexa e bastante singular, sendo o conceito de proteína ideal muito importante nesta conjuntura. Os aminoácidos ou proteínas prioritárias e essenciais dentro do equilíbrio alimentar, com capacidade de manter o desenvolvimento e produção da ave, sem carências ou exageros, são denominados de proteína ideal (Cardoso e Tessari, 2015).

Namazu et al. (2008) mostraram que alguns aminoácidos são considerados padrão, como no caso da lisina, que pode servir como estimativa de exigência para os demais aminoácidos, pois o mesmo é priorizado durante a formação proteica. A correta disponibilização da lisina na dieta préinicial, momento este que apresenta uma maior necessidade nutricional, pode acarretar vários aspectos positivos, como melhor conversão alimentar e desenvolvimento de peito, corte este mais valorizado e demandado pelo exigente mercado consumidor. 
Mais um aminoácido com importante função é a arginina, cuja deficiência traz um aumento do acúmulo de gordura abdominal, retardando o crescimento, podendo causar diversos tipos de disfuncionalidades. Thon et al. (2010) chegaram a conclusão que pintos de 1 a 7 dias, possuem exigência de $1,36 \%$ de arginina em sua dieta diária. Porém, um fator muito comum que pode alterar essa exigência é a presença de lisina, visto que elevados teores deste aminoácido no alimento pode causar um aumento da necessidade de arginina, logo, a relação lisina:arginina deve ser mantida estável, para não dificultar o processo produtivo (Campestrini et al., 2010).

Pesquisadores como Fernandes et al. (2017) conseguiram mostrar que a utilização de diferentes fontes de lipídeos pode influenciar no comprimento do intestino. Chegando a conclusão que aos sete de vida, a disponibilização de óleo de peixe durante a alimentação foi mais positiva para esta característica quando comparado ao óleo de soja. A glicina, dentro desde contexto de lipídeos, é um aminoácido fundamental na fase inicial, pois sua suplementação traz a possibilidade de melhorar a digestão de gordura, portanto aumentando a energia metabolizável disponível para a ave.

Dentro da dieta avícola, as vitaminas são substâncias essenciais para o bom desenvolvimento corporal do animal. Sendo a vitamina $\mathrm{D}$ muito importante, pois participa do sistema imunológico em geral e do metabolismo do cálcio. No entanto, a vitamina $\mathrm{D}$ é encontrada em distintas formas, como $\mathrm{D}_{2}$ (ergocalciferol) e $\mathrm{D}_{3}$ (colecalciferol) que biologicamente são inativas, e para serem utilizadas pelas aves, são transformadas pelo seu próprio organismo, para forma ativa de 1,25- dihidroxicolecalciferol. Neste último formato, a vitamina D é capaz de melhorar o resultado da morfometria intestinal na fase préinicial, já que foi capaz de gerar aumento das vilosidades intestinais e por consequência melhor absorção de nutrientes (Guerra et al., 2014).

Namazu et al. (2008) ainda apresentaram que substâncias como o zinco, são de fundamental importância no metabolismo de carboidratos, lipídeos e proteínas das aves, além de participar de atividades de aproximadamente 300 enzimas. $O$ mesmo está intimamente ligado ao crescimento de tecidos, auxilia o sistema imune, é essencial para divisão celular, participa da síntese proteica e de DNA, portanto, é um mineral primordial dentro da dieta animal.
Alguns outros elementos componentes das rações podem alterar o desempenho esperado das aves na fase pré-inicial, como no caso do sódio, quando disponibilizado entre a faixa de $0,25 \mathrm{e}$ $0,36 \%$ pode acarretar em um aumento do consumo de ração, melhorando o ganho de peso e favorecendo o rápido aumento de moela, intestino delgado e pró-ventrículo. Contudo, quando oferecido a altas taxas, como $0,48 \%$ causa efeito inverso, ocasionando queda no consumo de alimentos pelos pintos (Villanueva et al., 2015).

\section{Desenvolvimento trato digestório e digestibilidade dos nutrientes}

Em meio aos vários sistemas do organismo animal, provavelmente, o sistema digestório é o mais pesquisado e estudado. Já que ele é o principal responsável pelo ganho de peso das aves, sendo esta, a principal finalidade de todo ciclo produtivo. Assim, para que este sistema funcione de forma plena, deve-se conhecer os possíveis fatores que influenciam o desenvolvimento morfofuncional do mesmo (Lemos et al., 2016).

A partir do $17^{\circ}$ dia de incubação até o sétimo dia após o nascimento, as aves sofrem diferenciações morfológicas na anatomia do trato gastrointestinal, sobretudo, o acréscimo da quantidade de enterócitos, células epiteliais com função de absorção de nutrientes, e também com o aumento no tamanho do intestino (Santos et al., 2012).

Do ponto de vista prático, logo após o nascimento, o sistema digestório dos frangos está anatomicamente finalizado. Entretanto, sua competência funcional, como a capacidade de absorção e digestão de nutrientes ainda permanece imatura, quando confrontado com aves adultas (Maiorka et al., 2002).

As designadas células caliciformes, que possuem ocorrência nas criptas e vilosidades intestinais, são de fundamental importância para o bom desenvolvimento e conservação do sistema digestivo. Sendo que as mesmas são responsáveis pela secreção de substâncias estas encarregadas de proteger o epitélio contra o processo de digestão, auxiliando também na lubrificação e com melhora do trânsito alimentar no intestino (Rocha et al., 2016).

Após a eclosão, o trato gastrointestinal dos pintos é totalmente isento de micro-organismos, isso quando não acontece qualquer tipo de contaminação incomum durante a o período de incubatório ou fecundação, já que a 
industrialização do processo de incubação acabou por tornar impossível destes animais entrarem em contato com aves adultas, e começarem desenvolver precocemente a microbiota intestinal de proteção. Estudos apresentam que o intestino delgado somente será totalmente colonizado após duas semanas de vida, pelo contanto com ambiente que contenha microrganismos e pela alimentação das aves (Figueira e Andrade, 2014).

No transcorrer do tempo em que o alimento percorre todo o sistema digestório, determinadas bactérias, como do gênero Lactobacillus spp., especializadas em auxiliar no processo digestivo acabam atuando. A origem destes microrganismos muitas vezes é a própria cama de criação, principalmente, por causa de sua excessiva reutilização, transferindo suas características de um lote para outro. Podendo, não só expor as aves a organismos benéficos, como também agentes patogênicos (Oakley et al., 2014).

No contexto de absorção de nutrientes, a microbiota intestinal também exerce grande influência. Os microrganismos presentes no sistema digestório, podem sofrer alterações por diversos fatores, como tipo de processamento da ração, densidade de nutrientes e mudanças na composição das dietas. $\mathrm{E}$ com o frequente lançamento de novos produtos, como suplementos e aditivos destinados para criação de frangos, são necessários estudos específicos para conhecer as consequências positivas ou negativas, decorrente da utilização dos mesmos (Santos et al., 2012b).

Quando os micro-organismos convivem de forma equilibrada no organismo do animal, podem trazer diversos benefícios, como inibição do desenvolvimento de organismos patogênicos, possibilita uma melhor resposta imunológica da ave e auxilia na formação de algumas vitaminas. Mas caso estes micro-organismos estejam em desequilíbrio, podem acarretar sérios danos, desde a ocorrência de diarreias, queda na absorção de nutrientes, até putrefação intestinal (Borsatti et al., 2016).

Além da alimentação e microbiota, as condições de criação dos animais também acabam por influenciar algumas características do sistema digestório. Marchini et al. (2009), concluíram que aves criadas em ambiente com maiores temperaturas, exibiram menor comprimento dos vilos intestinais e menor profundidade das criptas, se comparados aos animais criados em ambiente com termoneutralidade. Por consequência, também obtiveram menor peso corporal aos 42 dias de idade, possivelmente por causa da queda no apetite, alta quantidade de energia utilizada para termorregulação, menor ingestão do alimento.

\section{Incubação de estágio único versus estágio múltiplo}

Antes de serem transportados para $\mathrm{o}$ incubatório, os ovos permanecem estocados na chamada sala de ovos, neste ambiente se procura oferecer características climáticas ideais para manter paralisado o desenvolvimento dos embriões, o denominado "zero fisiológico". Este processo é realizado buscando preparar o ovo para incubação e para evitar a mistura entre lotes, tendo como tempo de duração ideal, o máximo de três dias para o período de armazenamento, para que não ocorra queda nos índices zootécnicos (Nazareno et al., 2014).

No incubatório chegam ovos fecundados, com o embrião em pleno crescimento, sendo estes também chamados de ovos incubáveis, e os mesmos serão armazenados em condições ideais, e com especial atenção no controle em relação a temperatura, umidade e ventilação. Atento a todas estas características, será possível proporcionar aos embriões uma ótima modificação biológica e oferecer um produto final (pintos) de alta qualidade e rentabilidade aos granjeiros (Baracho et al., 2010; Rufino et al., 2014).

Dentre algumas variáveis, a temperatura de incubação deve ser observada, já que a mesma afeta diretamente a eclodibilidade dos pintos, sendo a temperatura de 37 a $38^{\circ} \mathrm{C}$ considerada ideal para os ovos e, qualquer alteração, mínima que ocorra desta variável pode gerar grades perdas (Gigli et al., 2009).

Os profissionais responsáveis pelo processo de incubação também devem ficar atentos à manutenção da umidade relativa nas incubadoras, que deve ficar entorno de 50 e $58 \%$, tanto para ovos oriundos de matrizes pesadas, velhas e jovens. Caso esta umidade fique muito abaixo do ideal pode ocorrer desidratação nos embriões, dificultando a eclosão e dando origem a animais pequenos e muito sensíveis. Se o contrário acontecer, ou seja, umidade muito alta poderá acarretar antecipação da eclosão, e em alguns casos, ocorre nascimento de animais sem o completo desenvolvimento (Barbosa et al., 2015).

Outra variável que pode comprometer o êxito do processo de incubação é relação entre as concentrações de $\mathrm{O}_{2}$ e $\mathrm{CO}_{2}$, também conhecida como coeficiente de respiração, pois estes dois 
gases são essenciais para o apropriado crescimento do embrião. As taxas ideais para o ambiente de incubação, são de números como o de $21 \%$ e $0,5 \%$ de $\mathrm{O}_{2}$ e $\mathrm{CO}_{2}$ respectivamente. Quando é aplicado aos ovos altos níveis de $\mathrm{CO}_{2}$, pesquisas demonstram que pode acarretar retardamento no crescimento do embrião (Macari et al., 2013).

Para assegurar um desenvolvimento embrionário de qualidade, além de fatores climáticos, a viragem dos ovos é muito relevante, pois procura evitar a aderência do embrião à casca do ovo, proporciona o correto acréscimo das membranas extraembrionárias, facilita a translocação de nutrientes e busca melhorar o fluxo de ar, assim, diminuindo a taxa de mortalidade e eclosão de pintos com má formação (Santana et al., 2014).

Neste período, Cardoso et al. (2009) descrevem a necessidade de serem estabelecidos alguns cuidados em relação a proliferação de microrganismos. Já que a contaminação durante a incubação pode acarretar diversos problemas, e expor as aves recém-eclodidas a diversos microrganismos, em um momento onde os mesmos estão mais susceptíveis a colonização do trato gastrointestinal. Isso pode causar infecções ao saco da gema e colisepticemia, demostrando assim, a importância de um manejo sanitário adequando e eficaz durante este período.

No processo de incubação, as máquinas incubadoras de estágio múltiplo são aquelas capazes de receber em um mesmo momento, ovos com embriões em diferentes idades. Neste tipo de incubadora, não é possível oferecer as temperaturas ideais para as diferentes fases de desenvolvimento destes fetos. O calor gerado pelos embriões mais velhos é utilizado para aquecer os mais novos e assim manter a temperatura da casca destes por volta de 37 a $38^{\circ} \mathrm{C}$, para que não ocorra queda na taxa de eclodibilidade (Molenaar et al., 2010).

As incubadoras de estágio único se caracterizam por receberem somente ovos em uma mesma fase de crescimento embrionário. Alguns autores, como Villanueva et al. (2016) mostram que máquinas com essa característica são capazes de fornecer as condições climáticas ideais a todos os embriões, fazer um melhor controle sanitário, facilitar a limpeza das máquinas, já que todos os ovos são retirados em um mesmo momento. Por consequência, é capaz de melhorar vários índices produtivos. Do ponto de vista ambiental, essas incubadoras sofrem algumas críticas, por utilizarem uma maior quantidade de energia elétrica quando comparadas as máquinas de estágio múltiplo, já que não utilizam do calor gerado pela diferença de idade entre os ovos.

\section{Considerações Finais}

São várias as necessidades e desafios para tornar toda cadeia produtora de aves cada vez mais competitiva, eficiente, e que sempre apresente um crescimento baseado na sustentabilidade. Consequentemente, para chegarmos a essa realidade, um melhor conhecimento sobre a nutrição e incubação se mostra imperativo.

Pesquisar e buscar de forma permanente o aperfeiçoamento de novas tecnologias, fazendo com que a alimentação e incubação do animal seja cada vez mais eficiente, aproveitando ao máximo o alimento, sendo este disponibilizado de acordo com suas diferentes fases de crescimento, dirimindo o desperdício e ininterruptamente respeitando o bem-estar-animal.

\section{Referências}

ABPA. Associação Brasileira de Proteína Animal. Relatório Anual 2018. Disponível em: <http://abpabr.com.br/storage/files/versao _final_para_envio_digital_1925a_final_abpa _relatorio

_anual_2018_portugues_web1.pdf> Acesso em: 01 ago. 2018.

Andrade, R.C.; Lara, L.J.C.; Pompeu, M.A.; Cardeal, P.C.; Miranda, D.J.A.; Baião, N.C. Avaliação da correção da energia pelo balanço de nitrogênio em alimentos para frangos de corte. Arquivo Brasileiro de Medicina Veterinária e Zootecnia, 68(2): 497-505, 2016.

Andrade, E.C.; Baião, N.C.; Lara, L.J.C.; Rocha, J.S.R.; Brumano, G.; Saldanha, M.M.; Abreu, A.R.C. Efeitos da granulometria e da forma física da ração sobre o desempenho de frangos de corte. Arquivo Brasileiro de Medicina Veterinária e Zootecnia, 68(2) 483-488, 2016b.

Baracho, M.S.; Nããs, I.A.; Gigli, A.C.S. Impacto das variáveis ambientais em incubatório de estágio múltiplo de frangos de corte. Engenharia Agrícola, 30 (4): 563-577, 2010.

Barbosa, V.M.; Baião, N.C.; Lara, L.J.C.; Rocha, J.S.R.; Pompeu, M.A.; Martins, N.R.S.; Leite, R.C.; Cançado, S.V. Efeitos da umidade relativa do ar na incubação e da idade da matriz leve sobre a eclodibilidade, qualidade dos pintos recém-eclodidos e desempenho da 
progênie. Arquivo Brasileiro de Medicina Veterinária e Zootecnia, 67(3): 882-890, 2015.

Boemo, L.S.; Rosa, D.P; Rosa, A.P.; Orso, C.; Scher, A.; Gehrke, S.B.; Sila, A.E.; Mariani, A.B. Processos térmicos em dietas para frangos de corte na fase pré-inicial. Revista Brasileira de Saúde Produção Animal, 17(2): 195-201, 2016.

Borsati, L.; Nunes, R.V.; Schone, R.A.; Frank, R.; Schneiders, J.L.; Savoldi, T.L. Digestibilidade de nutrientes em rações de frangos de corte suplementadas com promotores de crescimento. Arquivo Brasileiro de Medicina Veterinária e Zootecnia, 68(1): 201-207, 2016.

Campestrini, E; Barbosa, M.J.B; Nunes, R.V; Gasparino, E.; Silva, W.T.M.; Khul, R. Níveis de lisina digestível com dois balanços eletrolíticos para pintos de corte na fase inicial, de 1 a 21 dias de idade. Revista Brasileira de Zootecnia, 39(1): 151-157, 2010.

Cardeal, P.C.; Rocha, J.S.R.; Ferreira, H.C.; Santos, C.H.; Pompeu, M.A.; Cunha, C.E.; Baião, N.C; Lara, L.J.C. Efeito do transporte de péletes sobre sua qualidade. Arquivo Brasileiro de Medicina Veterinária e Zootecnia, 66(5): 1618-1622, 2014.

Cardoso, A.L.S.P.; Tessari, E.N.C.; Kanashiro, A.M.I.; Stoppa, G.F.Z.; Luciano, R.L.; Castro, A.G.M. Avaliação da qualidade sanitária de incubatórios por meio de placas de sedimentação. Arquivos do Instituto Biológico, 76(2): 279-283, 2009.

Cardoso, A.L.S.P.; Tessari, E.N.C. Interação entre imunidade e nutrição das aves: revisão de literatura. Revista Científica de Medicina Veterinária, 24(0), 2015.

Fernandes, J.I.M.; Rorig, A.; Gottardo, E.T.; Schimidt, J.M.; Burin Júnior, A.M.; Fulber, L.M. Dietas pós-eclosão suplementadas com fontes de gordura e acrescidas de taurina e glicina sobre a morfometria intestinal e o desempenho de frangos de corte de um a 21 dias. Arquivo Brasileiro de Medicina Veterinária e Zootecnia, 69(1): 198-204, 2017.

Figueira, S.V.; Andrade, D.M.A. Microbiota intestinal das aves de produção. Enciclopédia Biosfera, 2014.

Gigli, A.C.S.; Baracho, M.S.; Nããs, I.A.; Salgado, D.D.; Alvarenga, D.P. Environmental conditions in broiler multi-stage setter - a case study. Scientia Agricola, 66(2): 145-149, 2009.

Guerra, A.F.Q.G.; Murakami, A.E.; Santos, T.C; Eyng, C.; Picoli, K.P.; Ospinas Rojas, I.C. Utilização da vitamina D3 e seus metabólitos na alimentação de frangos de corte sobre parâmetros imunológicos e morfometria intestinal. Pesquisa Veterinária Brasileira, 34(5): 477-484, 2014.

Lara, L.J.C.; Campos, W.E.; Baião, N.C.; Lana, A.M.Q.; Cançado, S.V.; Rocha, J.S.R.; Pompeu, M.A.; Barbosa, V.M. Efeitos da forma física da ração e da linhagem de frangos de corte sobre a digestibilidade dos nutrientes e determinação de energia líquida. Arquivo Brasileiro de Medicina Veterinária e Zootecnia, 65(6): 1849-1857, 2013.

Lemos, M.J.; Calixto, L.F.L.; Torres Concordio, K.A.A.; Reis, T.L. Uso de aditivo alimentar equilibrador da flora intestinal em aves de corte e de postura. Arquivo do Instituto de Biológico, 83: 1-7, 2016.

López, C.A.A.; Baião, N.C. Efeitos do tamanho da partícula e da forma física da ração sobre o desempenho, rendimento de carcaça e peso dos órgãos digestivos de frangos de corte. Arquivo Brasileiro de Medicina Veterinária e Zootecnia, 56(2): 214-221, 2004.

López, C.A.A.; Baião, N.C.; Lara, L.J.C.; Rodriguez, N.M; Cançado, S.V. Efeitos da forma física da ração sobre a digestibilidade dos nutrientes e desempenho de frangos de corte. Arquivo Brasileiro de Medicina Veterinária e Zootecnia, 59(4): 1006-1013, 2007.

Lorençon, L.; Nunes, R.V.; Pozza, P.C.; Pozza, M.S.S.; Appelt, M.D.; Silva, W.T.M. Utilização de promotores de crescimento para frangos de corte em rações fareladas e pletizadas. Acta Scientiarum Animal Science, 29(2): 151-158, 2007.

Macari, M.; Gonzales, E.; Patrício, I.S.; Naas, I.A.; Martins, P.C. Manejo da incubação. $3^{\mathrm{a}}$ ed. Jaboticabal: FACTA, 2013. p. 306-307.

Marchini, C.F.P.; Silva, P.L.; Nascimento, M.R.B.M.; Beletti, M.E.; Guimarães, E.C.; Soares, H.L. Morfometria da mucosa duodenal em frangos de corte submetidos à temperatura ambiente cíclica elevada. Arquivo Brasileiro de Medicina 
Veterinária e Zootecnia, 61(2): 491-497, 2009.

Melo, A.S.; Queiroz, J.P.A.F.; Oliveira, M.; Dias, F.K.D.; Fernandes, R.T.V.; Marinho, J.B.M.; Souza, R.F.; Filho, C.A.S.; Souza, A.O.V.; Arruda, A.M.V. Formas físicas de utilização de rações para aves. Publicações em Medicina Veterinária e Zootecnia, 10(2): 173-178, 2016.

Molenaar, R.; Reijrink, I.A.M.; Meijerhof, R.; Van Den Brand, H. Meeting Embryonic Requirements of BroilersThroughout Incubation: A Review. Brazilian Journal of Poultry Science, 12(3): 137-148, 2010.

Namazu, L.B.; Kobashigawa, A.E.; Albuquerque, R.; Schammass, E.A.; Takeara, P.; Trindade Neto, M. A. Lisina digestível e zinco quelado para frangos de corte machos: desempenho e retenção de nitrogênio na fase pré-inicial. Revista Brasileira de Zootecnia, 37(9): 1634-1640, 2008.

Nazareno, A.C.; Silva, I.J.O.; Vieira, A.M.C.; Vieira, F.M.C. Microclima, idade das matrizes e tempo de estocagem influenciando nas respostas produtivas de ovos férteis. Revista Brasileira de Engenharia Agrícola e Ambiental, 18(11): 1172-1178, 2014.

Oakley, B.B.; Lillehoj, H.S.; Kogut, M.H.; Kim, W.K.; Maurer, J.J.; Pedroso, A.; Lee, M.D.; Collett, S.R.; Johnson, T.J.; Cox, N.A. The chicken gastrointestinal microbiome. Federation of European Microbiological Societies Microbiol Lett, 360: 100-112, 2014.

Perobelli, F.S.; Betarelli Júnior, A.A.; Vale, V.A.; Cunha, R.G. Impactos econômicos do aumento das exportações brasileiras de produtos agrícolas e agroindustriais para diferentes destinos. Revista de Economia e Sociologia Rural, 55(02): 343-366, 2017.

Reis, T.L.; Calixto, L.F.L.; Alves, O.S.; Lemos, M.J.; Lima, M.F.; Torres Cordido, K.A.A.; Curvello, F.A.; Sousa, F.D.R. Uso de aditivos nas fases pré-alojamento e pré-inicial sobre o peso corporal e do trato digestório de pintos de corte. Revista Brasileira de Saúde Produção Animal, 16(4): 811-817, 2015.

Rocha, P.M.C.; Barros, M.E.G.; Evencio Neto, J. Análise morfométrica da parede intestinal e dinâmica de mucinas secretadas no jejuno de frangos suplementados com probiótico Bacillus subtilis cepa C3102.
Pesquisa Veterinária Brasileira, 36(4): 312316, 2016.

Rostagno, H.S.; Albino, L.F.T.; Donzele, J.L.; Gomes, P.C.; Oliveira, R.F.; Lopes, D.C.; Ferreira, A.S.; Barreto, S.L.T.; Euclides, R.F. Tabelas brasileiras para aves e suínos (composição de alimentos e exigências nutricionais). $3^{\text {a }}$ ed. Viçosa: Universidade Federal de Viçosa, 2011. p. 77-80.

Rufino, J.P.F.; Cruz, F.G.G.; Machado, N.J.B.; Brasil, R.J.M.; Pereira, P.A.M.; Farias, E.G. Processos de incubação artificial associados à aplicação de diferentes métodos reprodutivos em matrizes semipesadas. Revista Brasileira de Saúde Produção Animal, 15(3): 765-773, 2014.

Santana, M.H.M.; Givisiez, P.E.N.; Figueiredo Júnior, J.P.; Santos, E.G. Incubação: principais parâmetros que interferem no desenvolvimento embrionário de aves. Revista Eletrônica Nutritime, 11(2): 33873398, 2014.

Santos, F.R.; Oliveira, P.R.; Minafra, C.S.; Duarte, E.F.; Almeida, R.R.; Silva, W.J. Desenvolvimento digestivo e aproveitamento energético em frangos de corte. Publicações em Medicina Veterinária e Zootecnia, 6(18), Ed. 205, Art. 1373, 2012.

Santos, I.I.; Corção, G.; Kessler, A.M.; Laranjeira, V.S.; Lima, M.S. Microbiota ileal de frangos de corte submetidos a diferentes dietas. Revista Brasileira de Zootecnia, 41(3): 643647, 2012b.

Santos, F.R.; Stringhini, J.H.; Miranda, C.S.; Almeida, R.R.; Oliveira, P.R.; Duarte, E.F.; Silva, R.B.; Café, M.B. Formulação de ração para frangos de corte de crescimento lento utilizando valores de energia metabolizável dos ingredientes determinada com linhagens de crescimento lento e rápido. Arquivo Brasileiro de Medicina Veterinária e Zootecnia, 66(6): 1839-1846, 2014.

Stringhini, J.H.; Rezende, A.D.; Café, M.B.; Leandro, N.S.M.; Andrade, M.A. Efeito do peso inicial dos pintos e do período da dieta pré-inicial sobre o desempenho de frangos de corte. Revista Brasileira de Zootecnia, 32(2): 353-360, 2003.

Surek, D.; Majorka, A.; Dahlkel, F.; Opalinski, M.; Franco, S.G.; Krabbe, E.L. Uso de fitase em dietas de diferentes granulometrias para frangos de corte na fase inicial. Ciência Rural, 38(6): 1725-1729, 2008. 
Tavernari, F.C.; Bernal, L.E.P.; Rostagno, H.S.; Albino, L.F.T.; Vieira, R.A. Relação metionina + cistina / lisina digestível para frangos de corte cobb. Revista Ceres, 61(2): 193-201, 2014.

Teixeira, E.N.M.; Silva, J.H.V.; Costa, F.G.P.; Martins, T.D.D.; Givisiez, P.E.N.; Furtado, D.A. Efeito do tempo de jejum pós-eclosão, valores energéticos e inclusão do ovo desidratado em dietas pré-iniciais e iniciais de pintos de corte. Revista Brasileira de Zootecnia, 38(2): 314-322, 2009.

Thon, M.S.; Stringuini, J.H.; Jardim Filho, R.M.; Andrade, M.A.; Café, M.B.; Leandro, N.S.M. Níveis de proteína e de arginina digestível na ração pré-inicial de frangos de corte. Revista
Brasileira de Zootecnia, 39(5): 1105-1111, 2010.

Villanueva, A.P.; Cardinal, K.M.; Krabbe, E.L.; Penz Junior, A.M.; Ribeiro, A.M.L. Influência da via de fornecimento do cloreto de sódio água ou ração - em frangos de corte de um a sete dias de idade. Revista Brasileira de Saúde Produção Animal, 16(4): 865-873, 2015.

Villanueva, A.P.; Ebling, P.D.; Pontalti, G.C.; Ribeiro, A.M.L. Effect of incubation system on the development of intestinal villi, metabolism, and performance of one- to fortyday-old broiler chickens. Revista Brasileira de Zootecnia, 45(9): 524-531, 2016. 